\title{
A STUDY OF CLINICAL PROFILE OF DENGUE FEVER IN A TERTIARY CARE CENTRE
}

\author{
Kalpana Chandra' ${ }^{1}$ Praveen Kumar ${ }^{2}$, Kalyan Kumar Saha 3 , Amarendu Kumar ${ }^{4}$, Manish Shankar 5
}

${ }_{1}^{1}$ Assistant Professor, Department of Pathology, IGIMS, Patna, Bihar. ${ }^{2}$ Associate Professor, Department of General Medicine, IGIMS, Patna, Bihar.

3 Senior Resident, Department of General Medicine, IGIMS, Patna, Bihar.

${ }^{4}$ Senior Resident, Department of General Medicine, IGIMS, Patna, Bihar.

${ }^{5}$ Associate Professor, Department of TB and Chest, IGIMS, Patna, Bihar.

\section{BACKGROUND}

ABSTRACT

Dengue fever is the most common arbovirus disease in urban and semi-urban areas of tropical country. Over the past three decades, there is dramatic increase in number leading to unprecedented panic amongst the general population. Hence, this study was designed to characterise the clinical profile of the outbreak.

Aims and Objectives- To evaluate the clinical profile of the patient of dengue fever.

\section{MATERIALS AND METHODS}

All patients of age above 14 years admitted in general medicine ward at SRMS-IMS, Bareilly with acute febrile illness and positive IgM antibody were included for study.

\section{RESULTS}

Dengue fever affected commonly young males in monsoon to post monsoon season. Fever was present in all the cases followed by gastrointestinal symptom in $62 \%$ cases. Other clinical features were body ache/ joint pain, headache, bleeding manifestation, weakness, respiratory symptoms, rashes, pruritus and altered sensorium in $43 \%, 26 \%, 24 \%, 24 \%, 12 \%, 9 \%, 3 \%$ and $1 \%$ cases respectively. Thrombocytopenia was present in $93 \%$ cases and leucopenia in $40 \%$ cases. Altered liver function test was very common biochemical abnormality with raised SGOT, SGPT and bilirubin in 90\%, $75 \%$ and $10 \%$ cases respectively. Ascites was present in $27 \%$ cases and pleural effusion in $17 \%$ cases.

\section{CONCLUSION}

Dengue fever is an important acute febrile illness in tropical countries affecting predominantly young males. It is commonly associated with fever, headache, body ache, gastrointestinal symptoms, thrombocytopenia, leucopenia and transaminitis. Dengue with organ dysfunction is associated with high mortality.

\section{KEYWORDS}

Dengue, DHF, DSS.

HOW TO CITE THIS ARTICLE: Chandra K, Kumar P, Saha KK, et al. A study of clinical profile of dengue fever in a tertiary care centre. J. Evolution Med. Dent. Sci. 2018;7(16):1989-1992, DOI: 10.14260/jemds/2018/447

\section{BACKGROUND}

Dengue fever is the most common arbovirus disease worldwide caused by four antigenically related dengue viruses $1,2,3,4 .{ }^{[1]}$ It is found in tropical and subtropical regions, predominantly in urban and semi-urban areas and now spreading in rural areas. In India, high number of cases has been reported in urban, periurban and rural areas. The cases peak after monsoon.[2]

Over the past three decades, there is dramatic increase in cases of dengue fever. Dengue epidemics are becoming a regular threat every year leading to significant mortality, morbidity and generating unprecedented panic amongst the general population. Hence, this study was designed to characterise the clinical profile of the outbreak.

'Financial or Other Competing Interest': None.

Submission 08-03-2018, Peer Review 01-04-2018,

Acceptance 09-04-2018, Published 16-04-2018.

Corresponding Author:

Dr. Praveen Kumar

Kailash-501, Jalapur Height,

Near RPS Mahilla College,

Mansarovar Colony, RPS More,

Bailly Road, Dananpur, Patna-801503, Bihar.

E-mail: praveen_kmr_23@yahoo.co.in

DOI: $10.14260 /$ jemds $/ 2018 / 447$

\section{Aims and Objectives}

To evaluate the clinical profile of patients of dengue fever.

\section{MATERIALS AND METHODS}

Patients admitted in the general medicine ward at SRMS-IMS, Bareilly in our unit were studied from July 2013 to Dec 2013. All patients of age above 14 years with acute febrile illness and positive IgM antibody for dengue virus were taken as case.

Selected patients were subjected to a thorough clinical examination and investigations like complete haemogram, KFT, LFT, chest $\mathrm{x}$-ray, ECG and ultrasound of abdomen, dengue serology, antigen malaria, Widal, urine $\mathrm{R} / \mathrm{M}$ and $\mathrm{C} / \mathrm{S}$, blood C/S along with serum amylase and lipase where indicated.

\section{RESULTS}

We included 100 cases for this study. The patients were subjected to a thorough clinical examination, investigation and a structured proforma was filled for each case. Descriptive statistics were calculated. Numbers and percentages were enumerated for all categorical variables such as clinical findings, haematological investigations, 
biochemical investigations and radiological investigations. Results are summarised as follows:-

1. Sex-wise distribution- Out of 100 cases, 68 were males and 32 were females with male-to-female ratio of 2.1: 1 .

2. Age-wise distribution (Table-1)- Dengue was prevalent in all age groups with age ranges from 15 to 75 years. Commonest age group was 21 to 30, contributing 38\% followed by 41 to 50 .

\begin{tabular}{|c|c|}
\hline Age & Frequency in Percentage \\
\hline $11-20$ & 17 \\
\hline $21-30$ & 38 \\
\hline $31-40$ & 17 \\
\hline $41-50$ & 20 \\
\hline $51-60$ & 6 \\
\hline $61-70$ & 1 \\
\hline $71-80$ & 1 \\
\hline \multicolumn{2}{|c|}{ Table 1 } \\
\hline
\end{tabular}

3. Month-wise distribution (Table-2)- Maximum number of cases reported in September (59\%) followed by October (25\%) and August (13\%).

\begin{tabular}{|c|c|}
\hline Month & Frequency in Percentage \\
\hline July & 2 \\
\hline August & 13 \\
\hline September & 59 \\
\hline October & 25 \\
\hline November & 1 \\
\hline December & 0 \\
\hline \multicolumn{2}{|c|}{ Table 2 } \\
\hline
\end{tabular}

4. Clinical Features (Table-3)- Fever was present in all the cases followed by gastrointestinal symptom such as nausea, vomiting, loose motion and abdominal distension in $62 \%$ cases. Other clinical features were body ache/ joint pain, headache, bleeding manifestation, weakness, respiratory symptoms, rashes, pruritus and altered sensorium in $43 \%, 26 \%, 24 \%, 24 \%, 12 \%, 9 \%$, $3 \%$ and $1 \%$ cases respectively.

\begin{tabular}{|c|c|}
\hline Clinical Features & Frequency in Percentage \\
\hline Fever & 100 \\
\hline Body ache/ Joint pain & 43 \\
\hline Headache & 26 \\
\hline Gastrointestinal symptom & 62 \\
\hline Bleeding manifestation & 24 \\
\hline Respiratory symptom & 12 \\
\hline Pruritus & 3 \\
\hline Weakness & 24 \\
\hline Rashes & 9 \\
\hline Altered sensorium Table 3 \\
\hline \multicolumn{2}{|c}{} \\
\hline \multicolumn{2}{|c}{} \\
\hline
\end{tabular}

5. Bleeding manifestation- Bleeding manifestation was present in $24 \%$ cases. The commonest site of bleeding was gastrointestinal bleeding in $16 \%$ cases. Other bleeding manifestations were epistaxis (2\%), bleeding gums $(2 \%)$, haemoptysis $(2 \%)$, haematuria $(1 \%)$ and pervaginal bleed (1\%).

6. Gastrointestinal Manifestation- Gastrointestinal symptom was the second most common presentation next to fever in dengue. The most common gastrointestinal manifestation was nausea and vomiting in $34 \%$ cases followed by abdominal pain, loose motion and abdominal distension in $14 \%, 8 \%$ and $6 \%$ cases respectively.

7. Haematological parameter (Table-4)- Out of 100 cases 5 cases were having PCV more than 45,40 cases were having TLC less than $4000 /$ cumm and 93 cases were having platelet less than $100000 /$ cumm. 47 cases were having lymphocytic predominance in DLC. Severe thrombocytopenia (platelet $<20000 /$ cumm), moderate thrombocytopenia (platelet 20001 to $50000 / \mathrm{cumm}$ ) and mild thrombocytopenia (platelet 500001 to 100000 /cumm) were present in $19 \%, 36 \%$ and $38 \%$ cases respectively.

\begin{tabular}{|c|c|}
\hline Variable & Frequency in Percentage \\
\hline PCV $>45$ & 5 \\
PCV $<45$ & 95 \\
\hline TLC $>4000 /$ cumm & 60 \\
TLC $<4000 /$ cumm & 40 \\
\hline Lymphocyte more than & 47 \\
neutrophil & 19 \\
Platelet $<20000 /$ cumm & 36 \\
Platelet 21000 - 50000/cumm & 38 \\
Platelet 51 - 100000/cumm & 7 \\
Platelet $>100000 /$ cumm & Table 4 \\
\hline \multicolumn{2}{|c|}{} \\
\hline
\end{tabular}

8. Biochemical parameter (Table-5)- Altered liver function test was very common biochemical abnormality in dengue. High SGOT, high SGPT and high bilirubin were present in $90 \%, 75 \%$ and $10 \%$ cases. Abnormal kidney function test was present in one case.

\begin{tabular}{|c|c|}
\hline Variable & Frequency in Percentage \\
\hline Bilirubin $>1.2 \mathrm{mg} / \mathrm{dL}$ & 10 \\
\hline SGOT $>60 \mathrm{IU}$ & 90 \\
\hline SGPT $>60 \mathrm{IU}$ & 75 \\
\hline Abnormal KFT & 1 \\
\hline \multicolumn{2}{|c|}{ Table 5 } \\
\hline
\end{tabular}

9. Radiological finding (CXR and USG)- Radiologically ascites was present in $27 \%$ cases, pleural effusion in $17 \%$ cases, hepatomegaly in $7 \%$ cases, splenomegaly in $7 \%$ cases, thickened gall bladder wall in $7 \%$ cases and hepatosplenomegaly in $5 \%$ cases.

10. Out of 100 cases, 6 patients were having secondary infection commonly in respiratory system and urinary system. Bacteria isolated were staphylococcal, E. coli, Klebsiella and Acinetobacter.

11. Out of 100 cases 2 patients died and 1 patient left against medical advice. $1^{\text {st }}$ patient died was 43 years female presented with fever with bleeding manifestation. Investigation revealed $\mathrm{Hb} 14.3 \mathrm{gm} \%$, TLC $1600 \mathrm{cmm} / \mathrm{dL}$, platelet 15000/cumm, S. bilirubin $2.5 \mathrm{mg} / \mathrm{dL}$, SGOT 5168IU, SGPT 1989IU, S. creatinine $5.9 \mathrm{mg} / \mathrm{dL}$ and positive dengue serology.

$2^{\text {nd }}$ patient died was 38 years old female who presented with fever. She was giving history of diabetes mellitus and hypothyroidism. Her investigation revealed $\mathrm{Hb} 10 \mathrm{gm} / \mathrm{dL}$, TLC 16000/cumm, platelet 60000/cumm, S. bilirubin $1.7 \mathrm{gm} / \mathrm{dL}$, SGOT 5177IU, SGPT 2503IU, hepatosplenomegaly and positive dengue serology. 


\section{DISCUSSION}

We conducted a clinical study on 100 cases of dengue fever at SRMS-IMS, Bareilly from July to December in 2013. Dengue fever affected all age groups with age ranges from 15 to 75 years. Commonest age group was 21 to 30, contributing 38\% followed by 41 to 50 . Nandini Chatterjee et al, Yagnik $\mathrm{H}$ Chhotala et al and Kakarla Thota Kanugolu Murali Mohan et al were having similar observation with a common affection in the age group of 20-29, 20-29 and 21-40 respectively.[3,4,5] Whereas Rachel Daniel et al observed that the incidence was equally distributed in all the three age groups, viz. 12.30, 30.50 and $>50$ years. The patient's mean age was 42.6 (SD 20) years. ${ }^{[6]}$

In our study, out of 100 cases 68 were male and 32 were female with male-to-female ratio of 2.1: 1. Yagnik H Chhotala et al and Kakarla Thota Kanugolu Murali Mohan et al were having similar observation with male-to-female ratio of 1.94:1 and 1.66:1 respectively. ${ }^{[4,5]}$ Whereas Nandini Chatterjee et al and Rachel Daniel et al observed almost equal distribution in male and female with male-to-female ratio of 0.95:1 and 1.08:1.[1,6]

In our study, maximum number of cases were reported in September (59\%) followed by October (25\%) and August (13\%). Kakarla Thota Kanugolu Murali Mohan et al reported more patients in October $(40.47 \%)$ followed by September and November.[5]

In our study, fever was present in all the cases followed by gastrointestinal symptom in $62 \%$ cases. Other clinical features were body ache/ joint pain, headache, bleeding manifestation, weakness, respiratory symptoms, rashes, pruritus and altered sensorium in $43 \%, 26 \%, 24 \%, 24 \%$, $12 \%, 9 \%, 3 \%$ and $1 \%$ cases respectively. Bleeding manifestations were present in $24 \%$ cases and the commonest site of bleeding was gastrointestinal bleeding. Ascites was present in $27 \%$ cases and pleural effusion in $17 \%$ cases. Nandini Chatterjee et al observed fever, headache with retro-orbital pain, myalgia, gastrointestinal symptom, skin rashes, bleeding manifestation disorientation and seizure in $98 \%, 90 \%, 86 \%, 42 \%, 28 \%, 23 \%, 10.4 \%$ and $3.8 \%$ cases respectively. They also observed isolated hepatomegaly in $13.5 \%$ cases, hepatosplenomegaly in $9 \%$ and evidence of effusions either pleural or peritoneal or both in $43 \%$ cases.[3] Yagnik H Chhotala, Chetal M Suva observed fever, headache, myalgia and vomiting in 100\%, 98\%, 97\% and 49\% cases respectively. They observed that warning signs were present in $28 \%$ patients out of 100 which includes ascites (8\%), hypotension (5\%), pulse pressure $<20 \mathrm{mmHg}(5 \%)$, positive tourniquet test (5\%), pleural effusion (3\%), uncommon gall bladder wall oedema (2\%), icterus (1\%) and altered sensorium (1\%).[4] Rachel Daniel et al observed fever in $96.8 \%$, headache in $77.2 \%$, abdominal pain in $62.4 \%$, diarrhoea in $15.2 \%$, pruritus in $10.4 \%$, bleeding manifestations occurred in $15.2 \%$ and positive tourniquet in $33.67 \%$ cases. Ascites was detected in $12 \%$, hepatosplenomegaly in $9.2 \%$, pleural effusion in $13.2 \%$ and polyserositis in $10 \%$ patients. Disseminated intravascular coagulation was noted in $3.2 \%$ cases.[6] Kakarla Thota Kanugolu Murali Mohan et al observed fever in all the 84 cases. Body pains and myalgia were present in $45 \%$, bleeding manifestation in $30 \%$, backache in $25 \%$, arthralgia in $25 \%$, abdominal pain with vomiting in $25 \%$, headache $20 \%$, hepatomegaly $18 \%$, splenomegaly $15 \%$, retro-orbital pain in
$15 \%$, diarrhoea in $15 \%$, upper respiratory catarrh with dry cough in $15 \%$, hyperaemic rash all over the body in $10 \%$, petechial rashes in 5\% and breathlessness in $6 \%$, altered sensorium with neck stiffness in $2.38 \%$ cases and seizures in $1.19 \%$ case. Ascites was found in $20 \%$ and bilateral pleural effusions in $6 \%$ cases. ${ }^{[5]}$

In our 100 cases $5 \%$ cases were having PCV more than 45 , $40 \%$ cases were having TLC less than 4000 / cumm and $93 \%$ cases were having platelet less than 100000/cumm. $47 \%$ cases were having lymphocytic predominance in DLC. High SGOT, high SGPT and high bilirubin were present in $90 \%$, $75 \%$ and $10 \%$ cases. Abnormal kidney function test was present in one case. Rachel Daniel et al observed haemoglobin $>16 \mathrm{gm} \%$ in $6 \%$ cases, TLC $<4000 / \mathrm{cmm}$ in $40 \%$ patients and platelet $<100000 / \mathrm{cmm}$ in $90 \%$ patients. Bilirubin was above $2 \mathrm{mg} \%$ in $9.7 \%$ and aspartate aminotransferase (AST) was > $45 \mathrm{IU} / \mathrm{L}$ in 83.9\%.[5] Yagnik $\mathrm{H}$ Chhotala et al observed thrombocytopenia in $79 \%$ cases followed by leucopenia in $56 \%$ patients, PCV ( $\geq 45 \%$ ) in $14 \%$ patients and $\mathrm{S}$. creatinine ( $>1.2 \mathrm{mg} \%$ ) in $8 \%$ patients.[4] Nandini Chatterjee et al observed HCT $<45 \%$ in $86 \%$ cases, TLC $<4000 / \mathrm{cmm}$ in $32.7 \%$, platelet count of $<100000 / \mathrm{cmm}$ in $55.6 \%$. Aspartate aminotransferase (AST) was $>45 \mathrm{IU} / \mathrm{L}$ in $72 \%$ cases.[3] Kakarla Thota Kanugolu Murali Mohan et al observed low platelet in $76.19 \%$ cases, leukopenia in $36.90 \%$ cases, leukocytosis in $16.66 \%$ cases, raised haematocrit in $35 \%$ cases, raised liver enzymes aspartate aminotransferase and alanine aminotransferase in $69.04 \%$ cases, moderately raised bilirubin and alkaline phosphatase levels in 5.955 cases and moderately raised serum creatinine and BUN in $5.95 \%$ cases.[5]

Out of 100 cases 6 patients were having secondary infection, commonly chest urinary system. Bacteria isolated were staphylococcal, E. coli, Klebsiella and Acinetobacter.

Out of 100 cases 2 patients of dengue fever with complication and multiorgan dysfunction died. Nandini Chatterjee et al observed case fatality rate of $3.8 \%$ and the causes of death were intracranial haemorrhage and DIC.[3] Yagnik H Chhotala, Chetal M Suva observed mortality rate in $4 \%$ cases and Rachel Daniel et al observed mortality in 3.2\% cases.[4,6] Surangrat Pongpan et al observed dengue fever with low systolic blood pressure, low pulse pressure, signs of vasculopathy, hepatomegaly, high haematocrit level, high SGOT and SGPT and prolonged PT and PTT are poor prognostic indicator. ${ }^{[7]}$

\section{CONCLUSION}

Dengue fever is an important acute febrile illness in tropical countries in monsoon to post monsoon season. It affects all age groups with predominant affection to young males. Fever, headache, bodyache and gastrointestinal symptoms are common presentations. Thrombocytopenia, leucopenia and transaminitis are common laboratory findings. Mortality is associated with complicated dengue with organ dysfunction.

\begin{tabular}{|c|c|c|}
\hline Sl. No. & Abbreviations & Full Form \\
\hline 1 & Hb & Haemoglobin \\
\hline 2 & PCV & Packed cell volume \\
\hline 3 & HCT & Haematocrit \\
\hline 4 & TLC & Total leukocyte count \\
\hline 5 & DLC & Differential leukocyte count \\
\hline 6 & PT & Prothrombin time \\
\hline
\end{tabular}




\begin{tabular}{|c|c|c|}
\hline 7 & aPTT & $\begin{array}{c}\text { Activated partial } \\
\text { thromboplastin time }\end{array}$ \\
\hline 8 & SGOT & $\begin{array}{c}\text { Serum glutamic oxaloacetic } \\
\text { transaminase }\end{array}$ \\
\hline 9 & AST & Aspartate transaminase \\
\hline 10 & SGPT & $\begin{array}{c}\text { Serum glutamic pyruvic } \\
\text { transaminase }\end{array}$ \\
\hline 11 & ALT & Alanine transaminase \\
\hline 12 & KFT & Kidney function test \\
\hline 13 & LFT & Liver function test \\
\hline 14 & ECG & Electrocardiogram \\
\hline 15 & R/M & Routine and microscopy \\
\hline 16 & C/S & Culture and sensitivity \\
\hline 17 & CXR & Chest x-ray \\
\hline 18 & USG & Ultrasonography \\
\hline & \multicolumn{2}{|c|}{ Abbreviations } \\
\hline
\end{tabular}

\section{ACKNOWLEDGEMENT}

I take this opportunity to extend my gratitude and sincere thanks to all those who helped me to complete this study. I am highly thankful to Department of General Medicine, Pulmonology, Pathology, Biochemistry, Microbiology and Critical Care Medicine for providing me adequate facility which helped me to carry out this study. I owe great sense of indebtedness to Dean SRMS-IMS, Bhojipura, Bareilly for permitting me to carry out this study.

\section{REFERENCES}

[1] Yeolkar ME. Dengue. In: Munjal YP, edr in chief. API text book of medicine. 9th edn. New Delhi: Jaypee Brothers 2012: p. 1158-60.

[2] Park K. The dengue syndrome: Park's textbook of preventive and social medicine. 24th edn. Jabalpur: M/s Banarsidas Bhanot Publisher 2017: p. 261-71.

[3] Chatterjee N, Mukhopadhyay M, Ghosh S, et al. An observational study of dengue fever in a tertiary care hospital of Eastern India. Journal of the Association of Physicians of India 2014;62(3):224-7.

[4] Chhotala YH, Suva CM. A study of clinical profile of dengue fever in a tertiary care hospital of Jamnagar, Gujarat, India. Int J Res Med Sci 2016;4(10):4500-4.

[5] Mohan KTKM, Rao BB, Raghuram C, et al. A clinical study of dengue fever cases: NRI Medical College, Chinakakani, Andhra Pradesh, South India. International Journal of Medicine and Public Health 2015;5(4):367-71.

[6] Daniel R, Rajamohanan, Philip AZ. Study of clinical profile of dengue fever in Kollam, Kerala, India. Dengue Bulletin 2005;29:197-202.

[7] Pongpana S, Wisitwong A, Tawichasri C, et al. Prognostic indicators for dengue infection severity. Int J Clin Pediatr 2013;2(1):12-8. 